\title{
AN INEQUALITY WITH APPLICATIONS TO STATISTICAL ESTIMATION FOR PROBABILISTIC FUNCTIONS OF MARKOV PROCESSES AND TO A MODEL FOR ECOLOGY
}

\author{
BY LEONARD E. BAUM AND J. A. EAGON
}

Communicated by R. C. Buck, November 21, 1966

1. Summary. The object of this note is to prove the theorem below and sketch two applications, one to statistical estimation for (probabilistic) functions of Markov processes [1] and one to Blakley's model for ecology [4].

\section{Result.}

TheOREM. Let $P(x)=P\left(\left\{x_{i j}\right\}\right)$ be a polynomial with nonnegative coefficients homogeneous of degree $d$ in its variables $\left\{x_{i j}\right\}$. Let $x=\left\{x_{i j}\right\}$ be any point of the domain $D: x_{i j} \geqq 0, \quad \sum_{j=1}^{a_{i}} x_{i j}=1, i=1, \cdots, p$, $j=1, \cdots, q_{i}$. For $x=\left\{x_{i j}\right\} \in D$ let $\Im(x)=\Im\left\{x_{i j}\right\}$ denote the point of $D$ whose $i, j$ coordinate is

$$
J(x)_{i j}=\left(\left.x_{i j} \frac{\partial P}{\partial x_{i j}}\right|_{(x)}\right) /\left.\sum_{j=1}^{q_{i}} x_{i j} \frac{\partial P}{\partial x_{i j}}\right|_{(x)} .
$$

Then $P(J(x))>P(x)$ unless $J(x)=x$.

Notation. $\mu$ will denote a doubly indexed array of nonnegative integers: $\mu=\left\{\mu_{i j}\right\}, j=1, \cdots, q_{i}, i=1, \cdots, p . x^{\mu}$ then denotes $\prod_{i=1}^{p} \prod_{j=1}^{g_{i}} x_{i j}^{\mu_{i j}}$. Similarly, $c_{\mu}$ is an abbreviation for $c_{\left\{\mu_{i j}\right\}}$. The polynomial $P\left(\left\{x_{i j}\right\}\right)$ is then written $P(x)=\sum_{\mu} c_{\mu} x^{\mu}$.

In our notation:

$$
J(x)_{i j}=\left(\sum_{\mu} c_{\mu} \mu_{i j} x^{\mu}\right) / \sum_{j=1}^{q_{i}} \sum_{\mu} c_{\mu} \mu_{i j} x^{\mu} .
$$

We wish to prove

$$
P(x)=\sum_{\mu} c_{\mu} x^{\mu} \leqq \sum_{\mu} c_{\mu} \prod_{i=1}^{p} \prod_{j=1}^{q_{i}} J(x)_{i j}^{\mu_{i j}} .
$$

ProOF.

$$
\begin{aligned}
P(x)= & \sum_{\mu}\left\{c_{\mu} \prod_{i=1}^{p} \prod_{j=1}^{q_{i}} J(x)_{i j}^{\mu i j}\right\}^{1 / d+1} \\
& \times\left\{c_{\mu}^{d l^{d+1}} x^{\mu} \prod_{i=1}^{p} \prod_{j=1}^{q_{i}}\left(\frac{1}{J(x)_{i j}}\right)^{\mu_{i j} / d+1}\right\} .
\end{aligned}
$$


We apply Hölder's inequality $[6$, p. 21] to obtain

$$
\begin{aligned}
P(x) \leqq & \left\{\sum_{\mu} c_{\mu} \prod_{i=1}^{p} \prod_{j=1}^{q_{i}} J(x)_{i j}^{\mu_{i j}}\right\}^{1 / d+1} \\
& \times\left\{\sum_{\mu} c_{\mu} x^{\mu} \prod_{i=1}^{p} \prod_{j=1}^{q_{i}}\left(\frac{x_{i j}}{J(x)_{i j}}\right)^{\mu_{i j} / d}\right\}^{d / d+1} .
\end{aligned}
$$

(In the last braces we have used $\left(x^{\mu}\right)^{d+1 / d}=x^{\mu} \prod_{i=1}^{p} \prod_{j=1}^{q_{i}} x_{i j}^{\mu_{i j} / d}$.) Since $\sum_{i=1}^{p} \sum_{j=1}^{a_{i}} \mu_{i j} / d \equiv 1$ by homogeneity of $P$, we can apply the inequality of geometric and arithmetic means [6, p. 16] to the double products of the second brace of (3) to conclude:

$$
\sum_{\mu} c_{\mu} x^{\mu} \prod_{i=1}^{p} \prod_{j=1}^{q_{i}}\left(\frac{x_{i j}}{\Im(x)_{i j}}\right)^{\mu_{i j} / d} \leqq \sum_{\mu} c_{\mu} x^{\mu} \sum_{i=1}^{p} \sum_{j=1}^{q_{i}} \frac{\mu_{i j}}{d} \frac{x_{i j}}{\Im(x)_{i j}} .
$$

We now substitute the definition (1) of $J(x)_{i j}$ in the expression on the right of (4) and interchange the order of summation to obtain:

$$
\begin{aligned}
& \sum_{\mu} c_{\mu} x^{\mu} \sum_{i=1}^{p} \sum_{j=1}^{q_{i}} \frac{\mu_{i j}}{d} \frac{x_{i j}}{\Im(x)_{i j}} \\
&=\frac{1}{d} \sum_{\mu} c_{\mu} x^{\mu} \sum_{i=1}^{p} \sum_{j=1}^{q_{i}} \mu_{i j} x_{i j} \\
& \cdot\left(\sum_{j_{0}=1}^{q_{i}} \sum_{\mu^{\prime}} c_{\mu^{\prime}} \mu_{i j_{0}}^{\prime} x^{\mu^{\prime}}\right) /\left(\sum_{\mu^{\prime}} c_{\mu^{\prime}} \mu_{i j}^{\prime} x^{\mu^{\prime}}\right) \\
&=\frac{1}{d} \sum_{i=1}^{p} \sum_{j=1}^{q_{i}} x_{i j}\left[\left(\sum_{\mu} \mu_{i j} c_{\mu} x^{\mu}\right) /\left(\sum_{\mu^{\prime}} \mu_{i j}^{\prime} c_{\mu^{\prime}} x^{\mu^{\prime}}\right)\right] \\
& \cdot \sum_{j_{0}=1}^{q_{i}} \sum_{\mu^{\prime}} c_{\mu^{\prime}} \mu_{i j_{0}}^{\prime} x^{\mu^{\prime}} .
\end{aligned}
$$

For each $\langle i, j\rangle$ the expression within the brackets is $=1$ and by hypothesis for each $i, \sum_{j=1}^{a_{i}} x_{i j}=1$. Hence the whole last expression of (5) reduces to $(1 / d) \sum_{i=1}^{p} \sum_{j_{0}=1}^{a_{i}} \sum_{\mu^{\prime}} c_{\mu^{\prime}} \mu_{i j_{0}}^{\prime} x^{\mu^{\prime}}$. But this is just $(1 / d) \sum_{i j_{0}} x_{i j_{0}}$ $\cdot\left(\partial P / \partial x_{i j_{0}}\right)$ so by the Euler theorem for homogeneous functions it is equal to $\Sigma_{\mu} c_{\mu} x^{\mu}$.

Finally, if we use this upper bound $\Sigma_{\mu} c_{\mu} x^{\mu}$ for the expression within the second braces in (3), raise both sides of (3) to the $(d+1)$ st power, and divide both sides of the resulting inequality by $\left(\Sigma_{\mu} c_{\mu} x^{\mu}\right)^{d}$ we obtain the desired inequality (2).

That $P\left(J\left\{x_{i j}\right\}\right)>P\left\{x_{i j}\right\}$ if $\left\{x_{i j}\right\} \neq\left\{x_{i j}\right\}$ follows from (4) and the strictness of the inequality of geometric and arithmetic means if all summands are not equal. 
3. Application 1. The first application of this theorem is to statistical estimation for (probabilistically) lumped Markov chains. Let $S$ be the finite state space of a Markov chain. Let $f$ be a function from $S$ to $R$. Let $y \in R^{T}, T$ an integer, be an observation. In [1] the problem is considered of estimating the transition probabilities $a_{i j}$ for $i, j \in S$, given $y$.

Let $X=\left(f^{T}\right)^{-1}(y) . X \subseteq S^{T}$. For $x \in X, i, j \in S$, let $\nu_{i j}(x)$ be the number of times the pattern $\cdot, \cdot, \cdot, i, j, \cdot, \cdot, \cdot$ occurs in $x$. The function $P\left(\left\{a_{i j}\right\}\right)=\sum_{x \in X} \prod_{i, j \in S} a_{i j}^{\nu_{i j}(x)}$ may be interpreted as the "probability of observing $y$ given the transition probabilities $\left\{a_{i j}\right\}$." Note that $P$ is a homogeneous polynomial of degree $T$ with nonnegative (integer) coefficients in the variables $a_{i j}$.

An iterative procedure for estimating the transition probabilities $\left\{a_{i j}\right\}$ given $y$ is suggested in [1]. If $\left\{a_{i j}\right\}$ is an a priori estimate, let $A_{i j}^{\prime}=\left(\sum_{x \in X} \nu_{i j}(x) \prod_{k, l \in S} a_{k l}^{\nu_{k l}(x)} / P\left(\left\{a_{i j}\right\}\right) . A_{i j}^{\prime}\right.$ may be interpreted as the "a posteriori expected value of the frequency of transition from state $i$ to state $j$ given $y$ and the $a$ priori probabilities $\left\{a_{i j}\right\}$." Thus $A_{i j}^{\prime} / \Sigma_{j} A_{i j}^{\prime}$ may be thought of as an "a posteriori estimate of the transition probabilities given $y$." Since

$$
A_{i j}^{\prime} / \Sigma_{j} A_{i j}^{\prime}=a_{i j}\left(\partial P / \partial a_{i j}\right) / \Sigma_{j} a_{i j}\left(\partial P / \partial a_{i j}\right)
$$

by our theorem applied to the transformation $J\left\{a_{i j}\right\}=\left\{A_{i j}^{\prime} / \Sigma A_{i j}^{\prime}\right\}$ we conclude that $P\left(J\left\{a_{i j}\right\}\right) \geqq P\left(\left\{a_{i j}\right\}\right)$. In other words the $a$ posteriori estimate of transition probabilities increases the likelihood of the given observation $y$.

Various results on the convergence of hill climbing iteration procedures [2], [3], [5] may be adduced to show that for almost all starts successive iterations will converge to a connected component of the local maximum set of $P$. If $P$ has only finitely many local maxima then successive iterates converge to a point.

This is the usual case in the more general situation considered in [1] in which the observation $y_{t}$ at time $t$ is obtained from the Markov state $x_{t}$ at time $t$ according to $P\left(y_{t}=k \mid x_{t}=j\right)=b_{j k}$ where $b_{j k}$ is an $s \times r$ stochastic matrix which is also to be estimated. Here the identifiability problem does not arise since, according to a theorem of Ted Petrie [7], "in general" no other $\left(a_{i j}\right),\left(b_{j k}\right)$ yields the same $y$ probabilities as a given $\left(a_{i j}^{0}\right),\left(b_{j k}^{0}\right)$ (save for the $s$ ! relabellings of states).

The second application is to some results of Blakley and Dixon [2], [3], [4]. Let $\Gamma$ be a symmetric $p$-linear form on $R^{n}$ that has nonnegative coefficients with respect to the standard basis for $R^{n}$. Let $g(\eta)=\Gamma(\eta, \eta, \cdots, \eta)$ where $\eta$ is a vector in $R^{n}$. Since $g$ is then just a pth degree homogeneous polynomial with nonnegative coeff- 
cients of the components of $\eta$ we may apply the theorem of this note to it. In Blakley's model $g$ is the adaptation (rate of growth) of a population. The transformation in Blakley's model $\sigma(\eta)$ $=\eta_{i}\left(\partial g(\eta) / \partial \eta_{i}\right) / p q(\eta)$ is the same as the transformation $\Im\left\{x_{i j}\right\}$ where $x_{i j}=\eta_{j}, i=1, j=1, \cdots, n$.

In Blakley's model if $\eta$ is the distribution of genotypes at time $t$, then $\sigma(\eta)$ is the distribution at time $t+1$. Thus it follows from the theorem in this note with $i=1$ that adaptation is nondecreasing with time when evolution of the genotypes at a single locus is considered. Our theorem with $i>1$ yields the same conclusion under natural hypotheses for evolution of the genotypes at several loci. This nondecreasing of the adaptation with time is clearly a desirable feature of the model.

\section{REFERENCES}

1. L. E. Baum, A statistical estimation procedure for probabilistic functions of Markov processes, IDA-CRD Working Paper No. 131.

2. G. R. Blakley, Homogeneous non-negative symmetric quadratic transformations, Bull. Amer. Math. Soc. 70 (1964), 712-715.

3. G. R. Blakley and R. D. Dixon, The sequence of iterates of a non-negative nonlinear transformation. III, The theory of homogeneous symmetric transformations and related differential equations, (to appear).

4. G. R. Blakley, Natural selection in ecosystems from the standpoint of mathematical genetics, (to appear).

5. Wolfgang Hahn, Theory and application of Liapunov's direct method, PrenticeHall, Englewood Cliffs, N. J., 1963, pp. 139-150.

6. G. H. Hardy, J. E. Littlewood, and G. Polya, Inequalities, Cambridge Univ. Press, New York, 1959.

7. Ted Petrie, Classification of equivalent processes which are probabilistic functions of finite Markov chains, IDA-CRD Working Paper No. 181, IDA-CRD Log No. 8694.

Institute fOR DEFENSE ANALyses 